\title{
LA DUALIDAD SOCIAL EN LOS ESTUDIOS DE OPINIÓN EN PANAMÁ (OCTUBRE DE 2019 - FEBRERO DE 2021)
}

\author{
Jon Subinas ${ }^{1}$ \\ jsubinas@cieps.org.pa \\ Centro Internacional de Estudios Políticos y Sociales (CIEPS), Panamá
}

Panamá ha destacado por su acelerado crecimiento entre 2004 y 2018 lo que ha tenido como consecuencia que en 2017 pasará a formar parte de los países de renta alta, pero este éxito económico no ha conseguido revertir, ni corregir las altas tasas de desigualdad. Con la irrupción de la pandemia se han hecho visibles las consecuencias de las desigualdades estructurales preexistentes. Entre las expresiones más destacadas de la inequidad podemos señalar la dualidad social. Esta dualidad se materializa en la opinión pública en una gran fractura social con dos modelos de país y opiniones muy divergentes sobre el rol de lo público y las medidas a adoptar para hacer frente a la pandemia.

Palabras clave: desigualdad, dualidad social, opinión pública.

\section{SOCIAL DUALITY IN OPINION POLLS IN PANAMA (OCTOBER 2019 - FEBRUARY 2021)}

Panama has stood out for its accelerated growth between 2004 and 2018, which has had as a consequence that in 2017 it will become part of the highincome countries, but this economic success has not been able to reverse or correct the high rates of inequality. With the outbreak of the pandemic, the consequences of pre-existing structural inequalities have become visible. Among the most prominent expressions of inequity, we can highlight social duality. This duality materializes in public opinion in a great social fracture with two country models and very divergent opinions on the role of the public and the measures to be adopted to face the pandemic.

Keywords: inequality, social duality, public opinion.

1 Doctor en Sociología y Antropología por la Universidad Complutense de Madrid. 


\section{Introducción}

Panamá es un país conocido por su canal interoceánico y por sus servicios de intermediación financiera, pero es menos conocido por haber albergado una de las economías más exitosas del mundo. Entre el 2004 al 2018 Panamá tuvo un crecimiento promedio del 7.0\% frente al 3.3\% de América Latina (Astudillo, Fernández y Garcimartín 2019: 3). Este crecimiento se moderó en 2019, pero en 2020 fruto de la pandemia del Covid 19 Panamá, al igual que el resto de la región, ha transitado por un proceso de decrecimiento e incertidumbre. En el caso del país canalero este decrecimiento en 2020 ha sido especialmente acusado llegando al $-17.9 \%$, uno de los más altos del continente ${ }^{2}$. La pandemia ha hecho más evidentes algunas debilidades estructurales del modelo de desarrollo panameño vinculadas a las altas tasas de desigualdad.

En 2017, el Istmo pasó a formar parte de la lista de los países con ingresos altos, superando los $\$ 12,055$ de Ingreso Nacional Bruto (INB) per cápita, unos datos que le acercaban a países del sur de Europa. Este impresionante crecimiento no ha podido revertir la extrema desigualdad, pero con la irrupción de la crisis social, política y económica propiciada por la pandemia las inequidades sociales se han hecho más evidentes.

El "World Income Inequality Database (WIID)" analiza la distribución de ingresos en 189 países, con más de 11,000 data points y 3,500 observaciones únicas por país, el Istmo desde 1960 hasta 2018 figura en esta base de datos con altos índices de desigualdad (UNU-WIDER 2018), con un índice Gini ${ }^{3}$ de 0.498 en la medición de 2018 lo que constituye un registro muy alto. Se estima que entre 2019 y 2020 el índice Gini crecerá entre un 3\% y un 3.9\% (Bárcenas 2020: 19).

La desigualdad en Panamá no es algo coyuntural sino originario y constitutivo de la sociedad panameña. Según la obra del historiador panameño Alfredo Castillero Calvo, Panamá, gracias a su posición geográfica consiguió insertarse en la primera globalización, perpetuando esta posición en el tiempo. Este proceso ha posibilitado una gran circulación de bienes y mercancías en el país, pero ha tenido como contraparte una inequitativa distribución de la riqueza, siendo las élites coloniales, que una vez enriquecidas abandonaban el país, y las élites blancas de origen europeo, las principales beneficiadas de estos procesos históricos (Castillero Calvo 2018: 147-

2 Durante el 2020, la producción de bienes y servicios en la economía panameña, medida a través del PIB, presentó una caída de $-17.9 \%$, respecto al año anterior. Recuperado el 28 de abril de 2021 , de: https://www.inec.gob.pa/publicaciones/Default3.aspx?ID_PUBLICACION=1052\&ID_ CATEGORIA=4\&ID_SUBCATEGORIA=26.

3 El índice Gini mide la desigualdad en la distribución de ingresos siendo "0" la igualdad total (todos tienen los mismos ingresos) y "1" la máxima desigualdad (una persona tiene todos los ingresos y los demás ninguno). Los países más igualitarios suelen tener un índice entre 0.20 y 0.30 ; los más desigualitarios entre 0.4 y 0.5 (aproximadamente). 
166). En definitiva, el Istmo históricamente ha ido fijando un modelo favorecido por el comercio internacional pero que ha distribuido de forma muy desigual sus beneficios. La desigualdad incluye procesos históricos de largo plazo que han ido sedimentado la inequidad.

En términos territoriales, la zona de tránsito que alberga los entornos del Canal (comprendida por las provincias de Panamá, Colón y Panamá Oeste), tiene una exitosa e internacionalizada economía de servicios, la cara más visible del denominado "hub de las Américas" (Conniff y Bigler 2019), que concentra el 86.3\% del PIB (INEC 2019 b) y que agrupa al 59.4\% de la población (INEC 2019a), mientras que el resto de país, cuya economía es principalmente agropecuaria y turística, sufre profundas carencias en el acceso a recursos y bienes de primera necesidad. Pero esta desigualdad también está presente en la zona de tránsito, ya que, según un estudio del Ministerio de Economía y Finanzas del Gobierno de Panamá, el índice de Gini de la provincia de Panamá (que acapara gran parte de la actividad económica del país) ascendía a un 0.47 (MEF 2017: 48). Panamá es uno de los países menos equitativos del mundo dentro de una región que es la más desigual del mundo (CEPAL 2019:22).

En 2017 se creó el Centro Internacional de Estudios Políticos y Sociales (CIEPS) que tiene como misión analizar la realidad social y política de Panamá desde sus particularidades para compararlo con la región y a su vez pretende ser un acicate para dar un impulso a las ciencias sociales en el Istmo. CIEPS utiliza diferentes metodologías, priorizando la investigación por medio de encuestas. La primera encuesta presencial del CIEPS de 2019 tenía como objetivo analizar el desempeño democrático del país canalero desde un punto de vista social, económico y político por medio de una encuesta de opinión pública. En 2020 como consecuencia de la pandemia CIEPS no pudo realizar su segunda encuesta presencial, aunque sí realizó encuestas online. En los resultados de la primera encuesta se detectaron una serie de realidades y elementos estructurales que han condicionado el desempeño político y económico del país durante la pandemia, y se han visto reflejados en los resultados de las encuestas online durante la pandemia.

\section{La dualidad social como articulación de la desigualdad}

Se puede denominar sociedad dual a aquella sociedad en la que coexisten dos mundos en uno. Ambos tienen un gobierno, un territorio y una bandera comunes, aunque sus necesidades, modo de vida, inquietudes y educación son muy distintos.

A nivel económico en Panamá, El Canal y la zona de tránsito han desempeñado un papel dualizador en el modelo de desarrollo. Constituyendo la zona de tránsito el principal acaparador de capitales financieros con una economía de servicios internacionalizada frente al resto del país con una economía fundamentalmente agraria y turística con un acceso más limitado a estos capitales financieros. Por este motivo, Panamá es frecuentemente calificada como una economía dual (OCDE 2018: 3). 
En correspondencia con esta estructura productiva dual, Panamá presenta asimismo una estructura ocupacional altamente dualizada. Siguiendo datos de INEC, la proporción de la ocupación informal llegó al 52.8\% en septiembre de 2020 (INEC 2020).

En el informe "La matriz de la desigualdad en Panamá" se analiza cómo los trabajadores en el quintil de mayores ingresos casi un 90\% de los asalariados están afiliados a un sistema previsional, en cambio en el quintil de menores ingresos este porcentaje apenas llega al 32\%. Mientras que de los ocupados en las áreas rurales un $55.5 \%$ son catalogados como trabajadores por cuenta propia y familiares no remunerados, en las áreas urbanas esta categoría llega a menos de la mitad (25.5\%). Asimismo, en las áreas urbanas, un $35.3 \%$ se desempeña en actividades de baja productividad (Cecchini, Holz y Rodríguez Mojica 2020: 22).

En términos simbólicos y culturales, una de las narrativas que analizan las sociedades latinoamericanas en su relación de lo rural con lo urbano, se articula en torno a los conceptos de dualidad y concordancia. La concepción de la dualidad social (Lambert 1967; Tumín, 1971) entendería que existe una división que sitúa a los latinoamericanos en dos grandes polos, en dos sistemas de valores: uno "el urbano" que posee los valores de la clase media, el progreso y la modernidad, y otro polo, "el rural", que adopta los valores tradicionales, propios de la clase baja, más primitivos y erráticos. Frente a estos planteamientos, la teoría de la concordancia (Seligson 1972) adoptaría el modelo de la convivencia de lo rural y lo urbano, de lo moderno y de lo tradicional. La escuela de la concordancia apostaría por el fin de estas dualidades como lo hacen autores de la decolonialidad como Rivera Cusicanqui o De Souza Santos (Rivera Cusiqanqui y De Souza Santos 2013), entre otros muchos, con el objeto de avanzar en procesos de democratización y reconocimiento de la diversidad. Nestor García Canclini (2012) destaca la dimensión híbrida de las culturas, y según Peter Burke, América Latina es el espacio del hibridismo cultural par excellence.

Las asimetrías entre el campo y la ciudad generan valores y expectativas diferentes, algunas compartidas, y otras enfrentadas, también se dan muchos espacios híbridos entre "lo urbano y lo rural" y entre "la modernidad y la tradición", pero hay que destacar que los valores de la metrópoli y de los sectores con mayores recursos tienden a ser los valores predominantes.

Es definitiva, existen diferentes ejes estructurantes de la desigualdad como el nivel de ingresos, lo territorial (urbano o rural), el género, la etnia, el nivel de estudios, la situación sociolaboral, entre otros, que operan de forma solapada y cristalizan en diferentes dualidades.

En la encuesta presencial realizada por el CIEPS en 2019 estas dualidades se visibilizan en dos grupos: los trabajadores universitarios, mayoritariamente hombres, urbanos versus las personas encuestadas con estudios primarios, mayoritariamente mujeres rurales. En las encuestas en línea realizadas por CIEPS en 2020 y principios 
del 2021 estas dualidades han sido contrastadas entre los grupos de menores ingresos y los grupos de mayores ingresos.

Estas dualidades además de expresar divergentes opiniones hacen evidente la grave situación de vulnerabilidad que sufren algunos grupos y su gran desprotección social.

\section{Un gran angular sobre la sociedad panameña (2019-2021)}

Para analizar la sociedad dual vamos a utilizar dos fuentes de información: La Encuesta CIEPS de Ciudadanía y Derechos realizada en 2019 y las encuestas de opinión pública realizadas por el CIEPS en 2021.

La Encuesta CIEPS de Ciudadanía y Derechos cuenta con un extenso cuestionario con tres apartados, uno sobre economía, otro sobre política y otro sobre los valores y formación de la opinión pública. A través de la Encuesta de Ciudadanía y Derechos CIEPS se detectaron importantes debilidades y amenazas que condicionaron el desempeño del país frente a la pandemia.

\section{Tabla 1}

Ficha técnica Encuesta CIEPS de Ciudadanía y Derechos 2019

\begin{tabular}{|l|l|}
\hline $\begin{array}{l}\text { Institución que diseñó, } \\
\text { administró, analizó, ordenó y } \\
\text { pagó la encuesta }\end{array}$ & $\begin{array}{l}\text { Centro Internacional de Estudios Políticos y } \\
\text { Sociales (CIEPS) - AIP. }\end{array}$ \\
\hline Empresa que realizó la encuesta & Gallup Panamá \\
\hline $\begin{array}{l}\text { Procedimiento para seleccionar } \\
\text { las unidades muestrales }\end{array}$ & Muestreo aleatorio estratificado. \\
\hline Tamaño de la muestra & $\begin{array}{l}\text { Muestra 1: Nacional sin Darién ni comarcas } \\
\text { aledañas) 1,192 } \\
+ \\
\text { Muestra 2: Darién, Guna Yala y Emberá } \\
\text { Wounaan) 200 = 1,392. }\end{array}$ \\
\hline Ponderación & $\begin{array}{l}\text { Se suman las dos muestras aplicando un } \\
\text { coeficiente de ponderación en función del peso } \\
\text { poblacional de provincias y comarcas en el censo. }\end{array}$ \\
\hline Universo poblacional & Panameños/as y residentes extranjeros. \\
\hline Tamaño muestral & $\begin{array}{l}\text { Muestra 1: República de Panamá sin Darién, } \\
\text { Guna Yala, Emberá Wounaan. } \\
\text { Muestra 2: Darién, Guna Yala y Emberá } \\
\text { Wounaan. }\end{array}$ \\
\hline Técnica de recolección de datos & Entrevista cara a cara. \\
\hline $\begin{array}{l}\text { Fecha o periodo del trabajo de } \\
\text { campo }\end{array}$ & Del 11 al 24 de octubre de 2019. \\
\hline
\end{tabular}




\begin{tabular}{|l|l|}
\hline $\begin{array}{l}\text { Institución que diseñó, } \\
\text { administró, analizó, ordenó y } \\
\text { pagó la encuesta }\end{array}$ & $\begin{array}{l}\text { Centro Internacional de Estudios Políticos y } \\
\text { Sociales (CIEPS) - AIP. }\end{array}$ \\
\hline Margen de error calculado & $\begin{array}{l}\text { Muestra } 1:+/-2.8 \% . \\
\text { Muestra } 2:+/-6.9 \% . \\
\text { Para un nivel de confianza del } 95 \% \text { para ambas } \\
\text { muestras y representativo a nivel nacional. }\end{array}$ \\
\hline
\end{tabular}

Fuente: Elaboración propia a través de datos de la Encuesta CIEPS de Ciudadanía y Derechos 2019. CIEPS.

En cuanto a la estratificación social para identificar a los diferentes grupos en la encuesta se realizaron análisis de clúster por medio de tres variables socioeconómicas: el consumo, el nivel educativo y la situación sociolaboral.

\section{Tabla 2}

Variables parar crear los grupos sociales en la Encuesta CIEPS de Ciudadanía y Derechos.

\begin{tabular}{|l|l|}
\hline Nivel de "consumo" & $\begin{array}{l}\text { Número de artículos o servicios que tienen (rango de 0 a 14) } \\
\text { Entre los servicios y artículos se encuentran agua, agua } \\
\text { caliente, celular prepago, autos, motos, etc. }\end{array}$ \\
\hline $\begin{array}{l}\text { Nivel de educación } \\
\text { Formal }\end{array}$ & $\begin{array}{l}\text { Primaria (incompleta, completa y sin estudios), Secundaria } \\
\text { (incompleta y completa) y Universitaria } \\
\text { (incompleta y completa). }\end{array}$ \\
\hline Situación laboral & Trabaja, no trabaja, ama de casa, jubilado o pensionado. \\
\hline
\end{tabular}

Fuente: Elaboración propia a través de datos de la Encuesta CIEPS de Ciudadanía y Derechos 2019. CIEPS.

Desde las operaciones estadísticas de "clustering” surgieron 5 grupos:

- Grupo 1: Los que trabajan con estudios secundarios $(n=351)$

- Grupo 2: Los que trabajan con estudios universitarios $(\mathrm{n}=231)$

- Grupo 3: Los que trabajan o se dedican a labores domésticas con estudios primarios $(n=210)$

- Grupo 4: Los desempleados y las amas de casa $(\mathrm{n}=397)$

- Grupo 5: Los jubilados, pensionados y otros $(n=197)$ 
Tabla 3

Características principales de los 5 grupos sociales de la Encuesta CIEPS de Ciudadanía y Derechos.

\begin{tabular}{|c|c|c|c|c|c|}
\hline $\begin{array}{l}\text { Grupos/ } \\
\text { Características }\end{array}$ & Grupo 1 & Grupo 2 & Grupo 3 & Grupo 4 & Grupo 5 \\
\hline Educación & $\begin{array}{c}100 \% \text { con } \\
\text { estudios } \\
\text { secundarios }\end{array}$ & $\begin{array}{c}100 \% \text { con } \\
\text { estudios } \\
\text { universitarios }\end{array}$ & $\begin{array}{l}100 \% \text { con } \\
\text { estudios } \\
\text { primarios }\end{array}$ & $\begin{array}{c}72 \% \text { con } \\
\text { estudios } \\
\text { secundarios }\end{array}$ & $\begin{array}{l}47 \% \text { con } \\
\text { estudios } \\
\text { primarios }\end{array}$ \\
\hline Situación laboral & $100 \%$ trabaja & $100 \%$ trabaja & $\begin{array}{c}55 \% \text { trabaja } 45 \% \\
\text { ama de casa }\end{array}$ & $\begin{array}{l}49 \% \text { no trabaja } \\
51 \% \text { amas de } \\
\text { casa }\end{array}$ & $\begin{array}{c}70 \% \text { jubilados } \\
\text { pensionados, } \\
30 \% \text { no } \\
\text { trabaja }\end{array}$ \\
\hline Sexo & $67 \%$ hombre & $58 \%$ hombre & $58 \%$ mujer & $73 \%$ mujer & $63 \%$ hombre \\
\hline $\begin{array}{l}\text { Promedio de } \\
\text { consumo }\end{array}$ & 7.9 & 9.6 & 6 & 7.6 & 7.6 \\
\hline Región & $\begin{array}{l}\text { 61\% Panamá } \\
\text { Metropolitana }\end{array}$ & $\begin{array}{c}\text { 58\% Panamá } \\
\text { Metropolitana }\end{array}$ & $\begin{array}{c}67 \% \text { Resto del } \\
\text { país }\end{array}$ & $\begin{array}{c}\text { 61\% Panamá } \\
\text { Metropolitana }\end{array}$ & $\begin{array}{l}\text { 58\% Panamá } \\
\text { Metropolitana }\end{array}$ \\
\hline $\begin{array}{l}\text { Depende parcial } \\
\text { o totalmente de } \\
\text { alguien }\end{array}$ & $26 \%$ & $22 \%$ & $45 \%$ & $77 \%$ & $36.4 \%$ \\
\hline Etnia/Raza & $\begin{array}{l}43 \% \text { mestizo } \\
19 \% \text { blanco }\end{array}$ & $\begin{array}{l}46 \% \text { mestizo } \\
24 \% \text { blanco }\end{array}$ & $\begin{array}{l}40 \% \text { mestizo } \\
23 \% \text { indígenas }\end{array}$ & $\begin{array}{l}47 \% \text { mestiza } \\
16 \% \text { blanca }\end{array}$ & $\begin{array}{l}42 \% \text { mestizo } \\
22 \% \text { blanco }\end{array}$ \\
\hline $\begin{array}{l}\text { ¿Escuela } \\
\text { privada, } \\
\text { pública o } \\
\text { ambos? }\end{array}$ & $\begin{array}{l}84 \% \text { asistió a } \\
\text { escuela pública }\end{array}$ & $\begin{array}{l}65 \% \text { escuela } \\
\text { pública } \\
14 \% \text { escuela } \\
\text { privada } \\
21 \% \text { ambas }\end{array}$ & $\begin{array}{l}89 \% \text { escuela } \\
\text { pública }\end{array}$ & $\begin{array}{l}76 \% \text { escuela } \\
\text { pública } \\
15 \% \text { ambas }\end{array}$ & $\begin{array}{l}75 \% \text { escuela } \\
\text { pública } \\
11 \% \text { privada }\end{array}$ \\
\hline Religión & $\begin{array}{l}53.7 \% \text { católicos } \\
22 \% \text { protestante/ } \\
\text { evangélico }\end{array}$ & $\begin{array}{c}64 \% \text { católicos } \\
22 \% \\
\text { protestante/ } \\
\text { evangélico }\end{array}$ & $\begin{array}{c}48.3 \% \text { católicos } \\
38 \% \\
\text { protestante/ } \\
\text { evangélicos }\end{array}$ & $\begin{array}{c}52 \% \text { católicos } \\
34 \% \text { protestante/ } \\
\text { evangélico }\end{array}$ & $\begin{array}{c}69 \% \text { católicos } \\
22 \% \\
\text { protestantes/ } \\
\text { evangélico }\end{array}$ \\
\hline $\begin{array}{l}\text { Tenencia: } \\
\text { Dueño de } \\
\text { casa (hipoteca } \\
\text { pagada o por } \\
\text { pagar) }\end{array}$ & $30 \%$ & $25 \%$ & $40 \%$ & $34 \%$ & $43 \%$ \\
\hline
\end{tabular}

Fuente: Elaboración propia a través de datos de la Encuesta CIEPS de Ciudadanía y Derechos 2019. CIEPS.

En los resultados de la encuesta se pudo apreciar un gran contraste entre dos polos, "el grupo 2", trabajadores con estudios universitarios, 6 de cada 10 hombres, con un buen acceso a los bienes de consumo y residiendo 6 de cada 10 en la Ciudad de Panamá, y "el grupo 3", con un irregular acceso al mercado de trabajo, 6 de cada 10 mujeres, con niveles educativos primarios, la mayoría residiendo fuera de la Ciudad de Panamá y con el peor acceso a los bienes de consumo de todos los grupos. Estos dos polos catalizan lo que en términos teóricos hemos denominado dualidad social. 
Debido a las restricciones de la pandemia entre 2020 y a principios de 2021 no se pudo realizar una nueva encuesta presencial por lo que se optó por efectuar encuestas virtuales hasta que acabaran las restricciones por la pandemia.

Las encuestas online realizadas por CIEPS desde el comienzo de la pandemia pretenden medir el impacto social, económico y político de aquella con varias preguntas que se han repetido en diferentes ediciones (marzo 2020, junio 2020 y enero de 2021), lo que posibilita un análisis longitudinal. Además en febrero de 2021 se realizó un sondeo de opinión dirigida específicamente a medir la situación educativa durante la pandemia y la receptividad ciudadana frente a las diferentes modalidades de educación (presencial, semipresencial o a distancia). Las encuestas se difundieron mayoritariamente por medio de redes sociales, aunque también se utilizaron listados de WhatsApp.

La investigación online realizada tiene un enfoque fundamentalmente exploratorio adecuado para investigar nuevos fenómenos. La pandemia del coronavirus constituye un fenómeno sin precedentes, un nuevo contexto en el que miles de ciudadanos se encuentran confinados en sus casas por lo que una encuesta online constituye una herramienta apropiada para la coyuntura pandémica.

Los estudios online han conseguido contactar con diferentes perfiles por edad, género, nivel de ingresos y nivel de estudios. En edad y género sí se han conseguido unos estadísticos similares a los parámetros poblacionales, pero en el resto de los parámetros no. En torno a 8 de cada 10 encuestados que llenaron los cuestionarios lo hicieron desde Panamá y Panamá Oeste, las dos provincias más pobladas pero que aglutinan poco más del 50\% de la población. En las muestras la mitad de los encuestados contaban con unos ingresos mayores a 2000 dólares por hogar, y aproximadamente 8 de cada 10 contaban con estudios universitarios completos. Estos datos reducen mucho la representatividad de los resultados ya que generan una sobre representación de estos perfiles.

Gracias al empleo de muestreo "bola de nieve" se posibilitó ampliar el perfil con bajos estudios, menores ingresos, y las entrevistas de provincias y de comarcas indígenas. Pero en esta ocasión con las muestras disponibles no se pudo realizar un "clustering" para volver a diseñar los grupos sociales como en la Encuesta de Ciudadanía y Derechos. Esto último es debido a las limitaciones de los datos y las insuficiencias en las variables independientes, por lo que se decidió solo utilizar la variable de ingresos para estratificar las diferentes opiniones en las muestras. 
Tabla 4

Fichas técnicas de 4 encuestas online realizadas por CIEPS entre 2020 y principios de 2021.

\begin{tabular}{|c|c|c|c|c|}
\hline $\begin{array}{l}\text { Institución } \\
\text { que realizó la }\end{array}$ & \multicolumn{4}{|c|}{$\begin{array}{l}\text { Centro Internacional de Estudios Políticos y Sociales } \\
\text { (CIEPS) - AIP }\end{array}$} \\
\hline $\begin{array}{l}\text { Nombre de la } \\
\text { encuesta }\end{array}$ & $\begin{array}{l}\text { Encuesta virtual } \\
\text { sobre el impacto } \\
\text { del coronavirus en } \\
\text { el comportamiento } \\
\text { y la opinión } \\
\text { pública. }\end{array}$ & $\begin{array}{l}\text { Encuesta } \\
\text { virtual sobre } \\
\text { el impacto del } \\
\text { coronavirus en el } \\
\text { comportamiento } \\
\text { y la opinión } \\
\text { pública (II). }\end{array}$ & $\begin{array}{l}\text { Estudio de } \\
\text { opinión sobre } \\
\text { el impacto del } \\
\text { coronavirus en el } \\
\text { comportamiento } \\
\text { y la opinión } \\
\text { pública (III). }\end{array}$ & $\begin{array}{l}\text { Encuesta sobre } \\
\text { la educación } \\
\text { durante la } \\
\text { pandemia. }\end{array}$ \\
\hline $\begin{array}{l}\text { Procedimiento } \\
\text { para seleccio- } \\
\text { nar las unidades } \\
\text { muestrales }\end{array}$ & \multicolumn{4}{|c|}{ Muestreo no probabilístico } \\
\hline $\begin{array}{l}\text { Tamaño de la } \\
\text { muestra }\end{array}$ & $n=1,266$ & $\mathrm{n}=396$ & $\mathrm{n}=1,199$ & $\mathrm{n}=603$ \\
\hline $\begin{array}{l}\text { Universo } \\
\text { geográfico y } \\
\text { población }\end{array}$ & \multicolumn{4}{|c|}{ N= República de Panamá } \\
\hline $\begin{array}{l}\text { Técnica de } \\
\text { recolección de } \\
\text { datos }\end{array}$ & \multicolumn{4}{|c|}{ Entrevista online } \\
\hline $\begin{array}{l}\text { Fecha del trabajo } \\
\text { de campo }\end{array}$ & Marzo 2020 & Junio 2020 & Enero 2021 & Febrero 2021 \\
\hline $\begin{array}{l}\text { Margen de error } \\
\text { calculado }\end{array}$ & \multicolumn{4}{|c|}{$\begin{array}{l}\text { Al ser una encuesta no probabilística no se puede calcular el error } \\
\text { muestral. }\end{array}$} \\
\hline $\begin{array}{l}\text { Técnicas } \\
\text { complementarias } \\
\text { de muestreo }\end{array}$ & \multicolumn{4}{|c|}{$\begin{array}{l}\text { Muestreo "bola de nieve" contactando con organizaciones, } \\
\text { personas públicas y particulares para difundir la URL de la } \\
\text { encuesta. }\end{array}$} \\
\hline
\end{tabular}

Fuente: Elaboración propia a través de datos de las encuestas online 2020-2021. CIEPS.

En los datos de las encuestas online desagregados por nivel de ingresos se puede apreciar el mayor rango de contraste entre dos polos los segmentos de mayores ingresos versus los segmentos de menores ingresos, lo que da cuenta de la dualidad social. 


\section{Una sociedad desigual y desconfiada}

En la encuesta de Ciudadanía y Derechos del CIEPS realizada en octubre de 2019, siete de cada diez encuestados manifestaron que Panamá no es un país que ofrece igualdad de oportunidades a todos y a todas, dato que varió según cada grupo. En el caso de los grupos que trabajan con estudios universitarios este porcentaje asciende por encima del promedio, a un $73.6 \%$, que contrasta con el $64.3 \%$ de los encuestados con un nivel educativo más bajo. Los trabajadores universitarios tienden a manifestar un alto grado de crítica, tanto en la igualdad de oportunidades como en el funcionamiento del sector público, lo que pone de manifiesto el gran descontento de los profesionales con estudios terciarios en Panamá.

\section{Gráfico 1}

La igualdad de oportunidades en Panamá

¿Cree que Panamá ofrece las mismas oportunidades a todos?

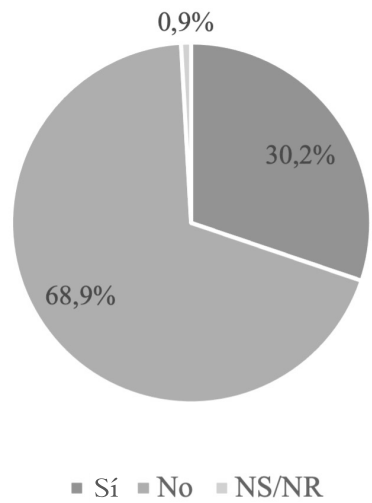

Fuente: Elaboración propia a través de datos de la Encuesta CIEPS de Ciudadanía y Derechos 2019. CIEPS.

Panamá es uno de los países más desiguales del mundo, dentro de una región que es la más desigual del planeta. Pero además de la disparidad de ingresos, que es la dimensión más determinante, la desigualdad tiene su correlato en distintas dimensiones manifestando otras inequidades sociales en el acceso a la educación, a la seguridad social, a la salud, al agua, al trabajo decente, es decir el fenómeno despliega una multiplicidad de factores. Unido a un aparato productivo y a un mercado laboral dual podemos destacar otras importantes fuentes de desigualdad como muestran los siguientes datos:

- En cuanto a déficits educativos, según la prueba PISA 2018, en Panamá solo un 35\% de los estudiantes alcanza un nivel mínimo de competencias en lectura, y un 19\% en matemáticas (De León 2019). Panamá ha tenido unos resultados 
demasiado bajos en lectura presentando el séptimo peor resultado en PISA 2018 y unos resultados nefastos en matemáticas con el tercer peor dato (Schleiler 2019: 6-7).

- Existen provincias como Bocas del Toro o Darién con numerosos sectores sin acceso a agua potable y en el caso de las comarcas indígenas esta deficiencia afecta a la mayoría de la población, además existe constancia de muchos sectores del país en los cuales el acceso al agua es irregular (Comité de Alto Nivel de Seguridad Hídrica 2016: 33-59).

- La falta de conectividad supone una grave brecha de inclusión social en la sociedad de la información. Según datos referidos en el Plan Nacional Estratégico de Ciencia, Tecnología e Innovación 2019-2024, solo un 11\% de la población cuenta con suscripciones de banda ancha fija, siendo mayoritarias las conexiones de banda estrecha por medio del celular. El 63\% de los hogares no tienen computadora; de los que no tienen computadora, el 71\% no usa internet (Urabarri 2020).

En el caso de la encuesta CIEPS de Ciudadanía y Derechos, un $80.9 \%$ piensa que la mayoría de gente en Panamá no es confiable "nunca o pocas veces". Un dato muy similar al Latinobarómetro 2018 en el que un $82.7 \%{ }^{4}$ de los encuestados en Panamá entiende que "uno nunca es suficientemente cuidadoso en el trato con las personas". La desconfianza se agudiza en sociedades con alta desigualdad como describe el sociólogo sueco Goran Therborn:

"Las ciencias sociales han comprobado de forma fehaciente que la desigualdad genera desconfianza (Uslaner 2002; Rothstein y Uslaner 2005) [...]. En los países escandinavos, dos tercios de la población creen que "la mayoría de la gente es confiable"; en Brasil solo el 3\% suscribe esta creencia, en Sudáfrica, el 12\%; en Gran Bretaña, el 30\%, y el Estados Unidos, el 36\% (Inglehart y Norris, 2004)" (Therborn 2013: 32).

Existen varias líneas de investigación que ratifican la correlación entre desigualdad y desconfianza como demuestran los trabajos de Bjornskov (2007: 1-21), Knack (1997: 1251-1288) y Cuartas Ricaurte (2016: 91-21).

Latinobarómetro 2018. Recuperado el 30 de abril de 2021, de: https://www.latinobarometro.org/ latContents.jsp 
Gráfico 2

Confianza interpersonal en Panamá

¿Se puede confiar en la gente en Panamá?

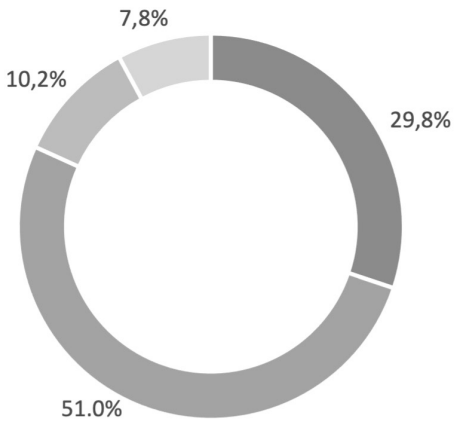

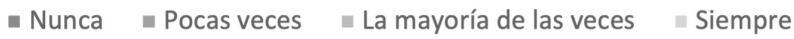

Fuente: Elaboración propia a través de datos de la Encuesta CIEPS de Ciudadanía y Derechos 2019. CIEPS.

\section{4. ¿Cómo solucionan los problemas económicos los panameños?}

Para resolver las dificultades económicas, los encuestados disponían en el cuestionario de nueve opciones: buscar un nuevo trabajo, pedir a un prestamista, utilizar sus ahorros, propiedades o inversiones, o bien acudir a sus redes más próximas, amigos, vecinos o familiares y, por último, solicitaban ayuda a líderes sociales, políticos o religiosos. Estas opciones las hemos agrupado en tres conjuntos:

1. El capital institucional: que incluye acudir a autoridades y liderazgos, sociales, políticos y religiosos. Este grupo abarca la ayuda solicitada a los líderes de la comunidad.

2. El capital social: que aglutina las redes sociales más cercanas, como los vecinos, los amigos o la familia. Este conjunto está relacionado con subespecies de capital social vinculados a las redes relacionales que se generan con los ámbitos más cercanos, los pares (Coleman 1988).

3. El capital económico: aglutina tres formas de solucionar problemas, buscar nuevas actividades profesionales, el capital financiero (ahorros, inversiones, préstamos de bancos o propiedades), o acudir a un prestamista en el caso de sufrir problemas económicos. 
Los datos señalan dos fuertes tendencias:

- Uno, que los grupos sociales que tienen una mejor posición en el mercado laboral, con mayor nivel educativo, son los que más acuden a los capitales económicos cuando tienen una dificultad.

- Dos, los grupos con un nivel educativo más bajo y peor situación sociolaboral tienen una menor propensión a utilizar los capitales económicos y una mayor predisposición a utilizar los capitales institucionales y sociales, acudiendo a familiares, amigos, líderes políticos locales y líderes religiosos cuando tienen un problema económico.

\section{Gráfico 3}

Distribución de tipos de capital por grupo 2 y 3

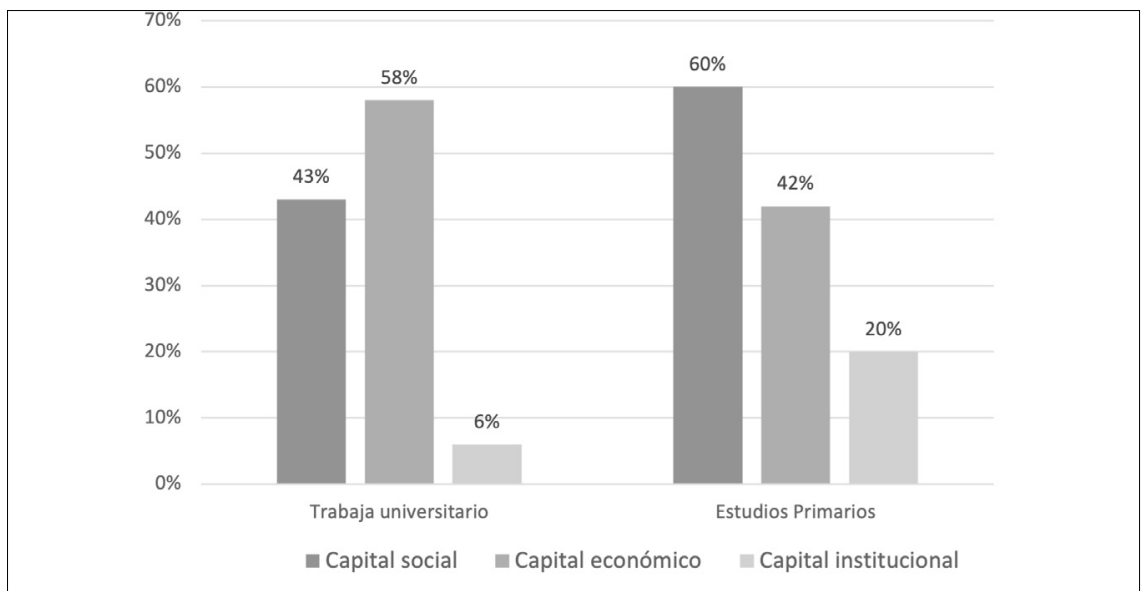

Pearson $\chi^{2}=\mathrm{p}<0.05$

Fuente: Elaboración propia a través de datos de la Encuesta CIEPS de Ciudadanía y Derechos 2019. CIEPS.

\section{Apuesta por lo público, pero con diferencias según el estatus social}

Con gran diferencia, las organizaciones que más desconfianza generan en el país son públicas y esenciales para el funcionamiento del régimen político. Solo el 25.3\% de las personas consultadas en 2019 por la Encuesta de Ciudadanía y Derechos tienen una opinión "buena" o "muy buena" sobre los partidos políticos; $38.3 \%$ sobre los sindicatos y $40.1 \%$ sobre el gobierno. Las mejores opiniones las generan organizaciones privadas y por definición verticales: la más alta es la de los medios de comunicación con $80.0 \%$; le siguen los bancos con $76.3 \%$; las pequeñas y medianas empresas con $71.5 \%$ y las iglesias católica y evangélica con $69.9 \%$ y $69.2 \%$, respectivamente. 
Esta percepción negativa de las instituciones concuerda con la visión de los servicios públicos. Estos servicios obtienen una evaluación negativa por parte de todos los encuestados, seis de cada diez tienen una valoración crítica. Existiendo una diferencia entre los que trabajan/universitario y los que tienen estudios primarios, las personas con estos estudios básicos expresan una mejor valoración de los servicios públicos.

\section{Tabla 5}

Calificación de los servicios públicos en Panamá

\begin{tabular}{ccccc}
\hline $\begin{array}{c}\text { Característica del } \\
\text { Grupo }\end{array}$ & $\begin{array}{c}\text { MUY MAL/ } \\
\text { MAL }\end{array}$ & $\begin{array}{c}\text { BIEN/ } \\
\text { MUY BIEN }\end{array}$ & NS/NR & Pearson $\boldsymbol{\chi}^{\mathbf{2}}$ \\
\cline { 1 - 4 } $\begin{array}{c}\text { Trabaja/Universitario } \\
\text { (Grupo 2) }\end{array}$ & $63.5 \%$ & $35.7 \%$ & $0.9 \%$ & $\mathrm{p}<0.05$ \\
\cline { 1 - 4 } $\begin{array}{c}\text { Estudios Primarios } \\
\text { (Grupo 3) }\end{array}$ & $53.1 \%$ & $42.6 \%$ & $4.3 \%$ & \\
\cline { 1 - 4 } Promedio & $61.8 \%$ & $35.7 \%$ & $2.5 \%$ & \\
\hline
\end{tabular}

Fuente: Elaboración propia a través de datos de la Encuesta CIEPS de Ciudadanía y Derechos 2019. CIEPS.

Si se analizan los resultados de la valoración de los servicios públicos por los diferentes sectores (educación, salud, agua, infraestructuras, electricidad, telecomunicaciones, vivienda, transporte, recogida de basuras y carreteras) se aprecian diferencias significativas entre los grupos.

Paradójicamente, los trabajadores universitarios que son los menos satisfechos con los servicios públicos de salud y educación, son los que menos los utilizan. Mientras que los que más los utilizan, el grupo de encuestados con menor nivel educativo, son los que expresan un menor nivel de crítica. Es importante destacar que, según la última encuesta del mercado laboral realizada por INEC en 2020, solo el 33\% de los encuestados de 20 años en adelante declaran tener educación universitaria, y solo el 22\% de los encuestados de todas las edades declaran disponer de educación universitaria (INEC 2020).

En la Encuesta de Ciudadanía y Derechos un 57.2\% de los trabajadores con estudios universitarios tienen una opinión muy negativa de los servicios públicos de educación mientras que en el grupo con estudios primarios esta opinión se reduce al $43.4 \%$. Se da la circunstancia que el $65 \%$ del grupo con estudios universitarios recibió su educación escolar en el sector público mientras que en el grupo de personas con estudios primarios ese porcentaje aumenta al $89 \%$.

En relación con los servicios públicos de salud, los trabajadores universitarios expresan un mayor grado de crítica, siendo un 78.4\% los que manifiestan opiniones negativas sobre el sector sanitario frente a un $58.0 \%$ de los encuestados con estudios primarios. Pero cuando en estos grupos tienen un problema de salud, aquellos con estudios primarios acuden a los servicios públicos hasta en un $64.3 \%$ de los casos 
mientras que los trabajadores con estudios universitarios solo lo hacen en el $37.7 \%$ de las ocasiones.

Las tendencias sobre quién debe proveer los diferentes servicios básicos son las siguientes:

1. Todos los encuestados entienden mayoritariamente que los servicios de educación, vivienda y sanidad debe proveerlos el sector público en exclusiva.

2. Pero dentro de los grupos se aprecian diferencias significativas. Aquellos que trabajan con estudios universitarios apoyan que los servicios básicos de educación, vivienda y salud los provea el sector público-privado, veintidós puntos más que grupo con menos estudios.

\section{Gráfico 4}

Provisión de servicios educativos por grupo de estudios primarios versus trabajadores universitarios

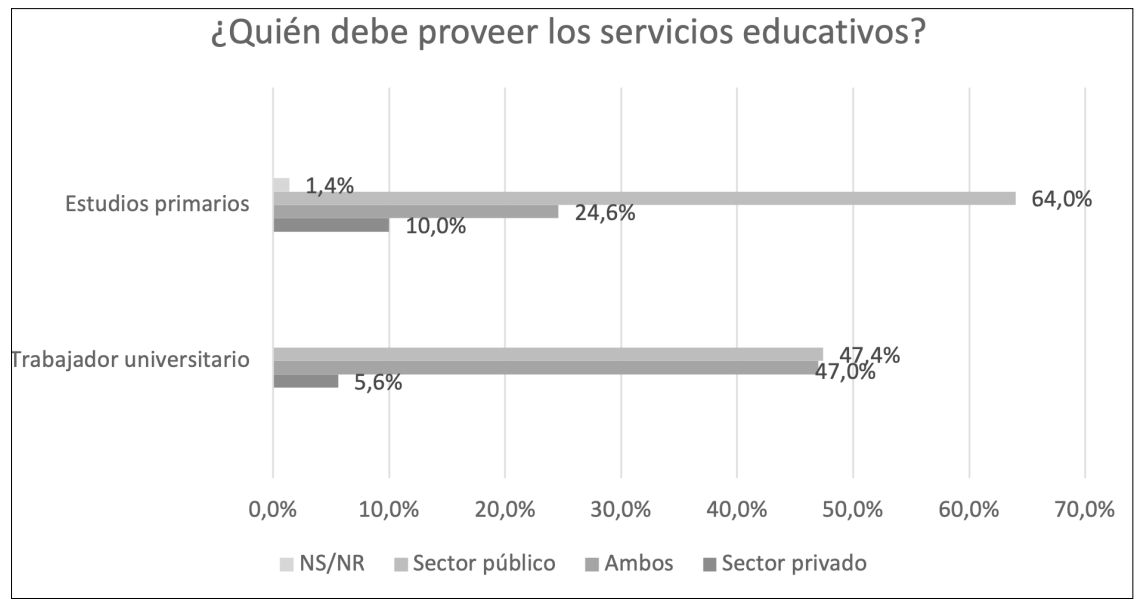

Pearson $\chi^{2}=p<0.05$

Fuente: Elaboración propia a través de datos de la Encuesta CIEPS de Ciudadanía y Derechos 2019. 


\section{Gráfico 5}

Provisión de servicios de vivienda por grupo de estudios primarios versus grupo trabajadores universitarios.

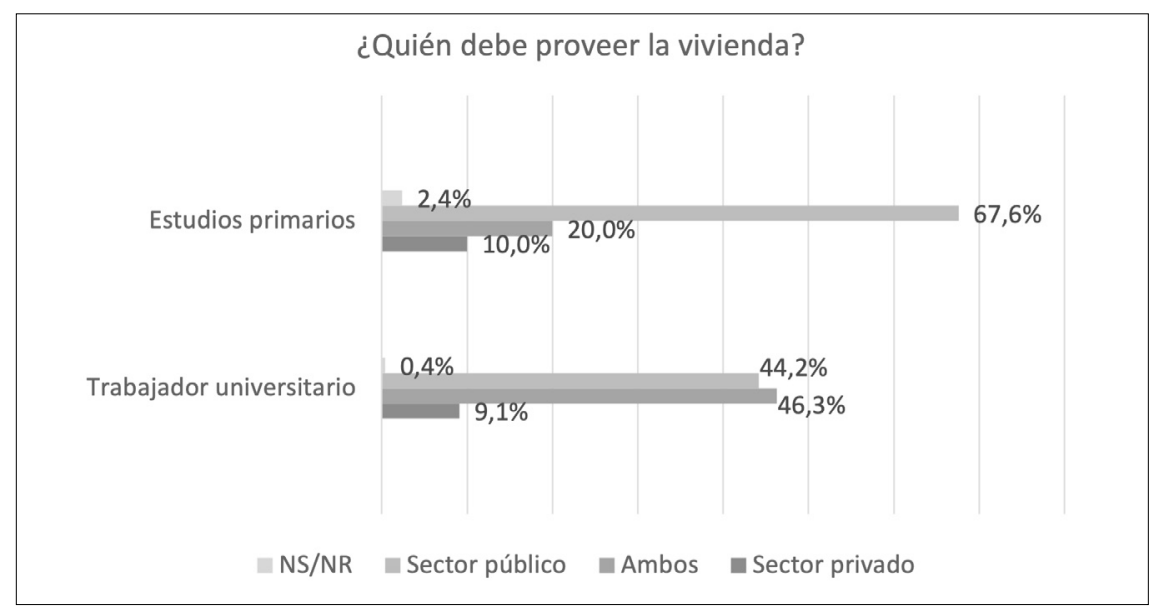

Pearson $\chi^{2}=\mathrm{p}<0.05$

Fuente: Elaboración propia a través de datos de la Encuesta CIEPS de Ciudadanía y Derechos 2019.

\section{Gráfico 6}

Provisión de servicios de salud por grupo de estudios primarios versus grupo trabajadores universitarios.

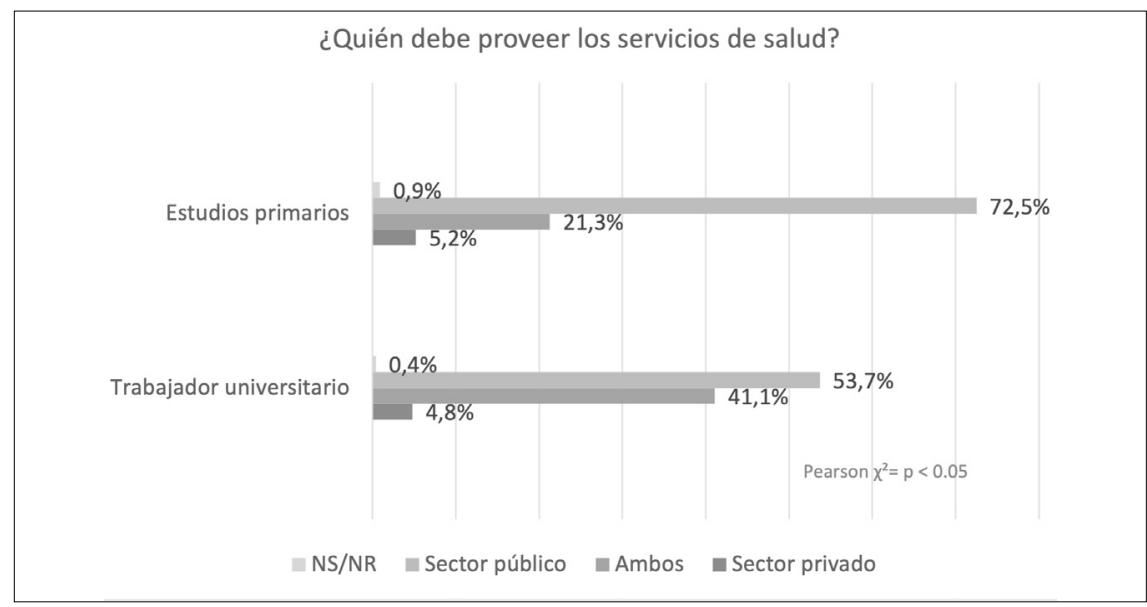

Pearson $\chi^{2}=\mathrm{p}<0.05$

Fuente: Elaboración propia a través de datos de la Encuesta CIEPS de Ciudadanía y Derechos 2019. 
Las personas que prefieren un modelo exclusivamente público tienden a tener una mejor percepción de Panamá como un país de oportunidades, usan los servicios públicos más frecuentemente, tienen una opinión más positiva sobre los servicios públicos que reciben, y tienden a depender más de sus contactos con instituciones como la iglesia, partidos políticos y los representantes de la administración pública. Por su parte, las personas que menos utilizan los servicios públicos y que valoran menos estos servicios tienen más predisposición a apostar por soluciones mixtas (público-privadas). En general, como comprobamos en el anterior apartado, este grupo tiende a depender más de su capacidad de crédito, ahorros y propiedades, además del acceso al mercado laboral para resolver sus problemas económicos.

Estos datos trazan una dualidad entre los grupos con mayores y menores recursos que apuntan hacia dos modelos de país que dificilmente van a encontrar soluciones consensuadas para hacer frente a los retos de la pandemia. Estas opiniones señalan indicios de dos modelos diferentes de concebir el rol del Estado. Una teoría sería más favorable a una solución público-privada para proveer los servicios básicos, esta corriente de opinión corresponde a los grupos con mayor capital económico y cultural (Bourdieu 1997), frente a los segmentos con menor capital económico y cultural que son más proclives a una solución exclusivamente pública.

\section{Y durante la pandemia, de nuevo la dualidad de opiniones}

El 9 de marzo de 2020 Panamá detectó su primer caso positivo de COVID-19 y cuatro días después declaró el estado de emergencia lo cual dotó al gobierno de poderes especiales para poder hacer frente a la pandemia. Esta rápida reacción fue muy bien acogida por la opinión pública. En marzo de 2020 en la "Encuesta virtual sobre el impacto del coronavirus en el comportamiento y la opinión pública" de CIEPS, la evaluación positiva del gobierno y del ministerio de salud superaba el $70 \%$. Esta evaluación se fue deteriorando por la evolución del virus que llegó a ser la más alta del continente a pesar de sufrir los confinamientos más extensos y severos de la región, por algunos escándalos en el manejo de los fondos públicos y por el agravamiento de la situación económica, quedando en un $11.9 \%$ los que aprueban la acción del gobierno y en $26.5 \%$ los que consideran positiva la acción del ministerio de salud en enero de 2021.

El 11 de mayo de 2020 el gobierno panameño anunció un proceso gradual de "desconfinamiento" dividido en seis bloques que tuvo que ser interrumpido abruptamente en junio por el crecimiento del número de casos de Covid-19. Unos días antes CIEPS realizó la "Encuesta virtual sobre el impacto del coronavirus en el comportamiento y la opinión pública (II)" que detectó diferencias ostensibles entre los grupos de ingresos sobre cuáles deberían ser los pasos que deberían tomar las autoridades con las cuarentenas y los confinamientos. Solo un $32.8 \%$ de los encuestados con 5,000 y más dólares consideran que el ritmo de desconfinamiento 
iba muy rápido mientras que en el resto de los grupos de ingresos prevalecían los que pensaban lo contrario, en especial las personas encuestadas con 2,000 o menos dólares de ingresos, entre los cuales un $71.9 \%$ en promedio pensaba que había que esperar.

\section{Tabla 6}

Evaluación de las medidas de desconfinamiento propuestas en mayo de 2020 por el gobierno panameño.

\begin{tabular}{|c|c|c|c|c|c|}
\hline & $\begin{array}{l}\text { Vamos muy } \\
\text { rápido hay que } \\
\text { esperar }\end{array}$ & $\begin{array}{l}\text { El ritmo que } \\
\text { se lleva es el } \\
\text { correcto }\end{array}$ & $\begin{array}{l}\text { Teníamos que haber } \\
\text { empezado antes }\end{array}$ & $\begin{array}{c}\text { No Sabe / No } \\
\text { Responde }\end{array}$ & Pearson $\chi^{2}$ \\
\hline 5,000 o más & $32.8 \%$ & $43.8 \%$ & $18.8 \%$ & $4.7 \%$ & \multirow[t]{6}{*}{$\mathrm{p}<0.05$} \\
\hline 3,001 a 4,999 & $62.3 \%$ & $22.6 \%$ & $15.1 \%$ & $0.0 \%$ & \\
\hline $\begin{array}{l}\text { Más de } 2,000 \text { a } \\
3,000\end{array}$ & $59.3 \%$ & $29.6 \%$ & $7.4 \%$ & $3.7 \%$ & \\
\hline $\begin{array}{l}\text { Más de } 1,000 \text { a } \\
2,000\end{array}$ & $77.2 \%$ & $8.9 \%$ & $12.7 \%$ & $1.3 \%$ & \\
\hline 400 a 1,000 & $69.6 \%$ & $22.8 \%$ & $7.6 \%$ & $0 \%$ & \\
\hline Menos de 400 & $69.0 \%$ & $7.1 \%$ & $19.0 \%$ & $4.8 \%$ & \\
\hline
\end{tabular}

Fuente: Elaboración propia a través de datos de la Encuesta virtual sobre el impacto del coronavirus en el comportamiento y la opinión pública (II). Junio 2020. CIEPS.

En la "Encuesta virtual sobre el impacto del coronavirus en el comportamiento y la opinión pública (II)" realizada por CIEPS en enero de 2021, en la pregunta si las personas estarían dispuestas a vacunarse cuando empezara el proceso, un $74.2 \%$ indicó que sí; otro 14\% señaló que no sabía; y solo un 11.8\% manifestó que no lo haría. Pero profundizando en las cifras se constataron diferencias significativas. Los resultados de la encuesta hicieron manifiesta una gran diferencia en la predisposición a vacunarse entre personas con ingresos superiores a 2,000 dólares frente a aquellos que tienen ingresos inferiores a los 400 dólares. La distancia entre estos dos perfiles socioeconómicos asciende a más de 20 puntos porcentuales en favor de los más acaudalados.

En el caso del "no sabe/no responde" las personas con menores ingresos fueron las que más utilizaron esta categoría de respuesta (22.2\%), lo que puede ser una fuente indicativa de dudas frente a la vacunación. Estas inquietudes se reducen a menos de la mitad en personas de más de 2,000 dólares de ingresos (10.6\%). 


\section{Tabla 7}

Predisposición a ponerse la vacuna contra el Covid 19

\begin{tabular}{|lc|c|c|c|}
\hline & Sí & No & No Sabe / No Responde & Pearson $\chi^{2}$ \\
Más de 2,000 & $82.4 \%$ & $7.0 \%$ & $10.6 \%$ & $\mathrm{p}<0.05$ \\
\cline { 1 - 3 } Más de 1,000 a 2,000 & $75.0 \%$ & $13.3 \%$ & $11.6 \%$ & \\
\cline { 1 - 3 } De 400 a 1,000 & $67.1 \%$ & $16.4 \%$ & $16.4 \%$ & \\
\hline Menos de 400 & $58.3 \%$ & $19.4 \%$ & $22.2 \%$ & \\
\hline
\end{tabular}

Fuente: Elaboración propia a través de datos de la Encuesta virtual sobre el impacto del coronavirus en el comportamiento y la opinión pública (II). Enero 2021. CIEPS.

En esta misma encuesta de enero de 2021 las personas con menos ingresos expresaron el máximo grado de desconfianza hacia las vacunas: un $52.8 \%$ confiaban poco o nada, frente al $21.3 \%$ de desconfianza entre quienes poseían ingresos que superaban los 2,000 dólares de forma mensual.

Tabla 8

Nivel de confianza en las vacunas anti Covid 19

\begin{tabular}{|c|c|c|c|c|c|c|}
\hline & $\begin{array}{c}\text { No confio } \\
\text { nada }\end{array}$ & Confio poco & $\begin{array}{l}\text { Confio } \\
\text { bastante }\end{array}$ & $\begin{array}{c}\text { Tengo mucha } \\
\text { confianza }\end{array}$ & $\begin{array}{c}\text { No Sabe / No } \\
\text { Responde }\end{array}$ & Pearson $\chi^{2}$ \\
\hline Más de 2,000 & $4.3 \%$ & $17.0 \%$ & $36.5 \%$ & $41.5 \%$ & $0.7 \%$ & \multirow[t]{4}{*}{$\mathrm{p}<0.05$} \\
\hline $\begin{array}{l}\text { Más de } 1,000 \text { a } \\
2,000\end{array}$ & $8.8 \%$ & $21.3 \%$ & $38.3 \%$ & $29.2 \%$ & $2.5 \%$ & \\
\hline De 400 a 1,000 & $12.0 \%$ & $29.3 \%$ & $33.8 \%$ & $23.6 \%$ & $1.3 \%$ & \\
\hline Menos de 400 & $18.5 \%$ & $34.3 \%$ & $25.9 \%$ & $18.5 \%$ & $2.8 \%$ & \\
\hline
\end{tabular}

Fuente: Elaboración propia a través de datos de la Encuesta virtual sobre el impacto del coronavirus en el comportamiento y la opinión pública (II). Enero 2021. CIEPS.

En concordancia con estos resultados en la "Encuesta sobre la educación durante la pandemia" realizada por CIEPS en febrero de 2021, aplicada al comienzo del curso escolar, se detectaron importantes diferencias de opinión entre los diversos grupos de ingresos. Es importante destacar que Panamá es uno de los países que más tiempo ha tenido cerradas las escuelas del mundo, esto da más relevancia a los resultados ${ }^{5}$.

Las personas encuestadas con ingresos inferiores a 400 dólares consideraban que la modalidad educativa más adecuada para el curso escolar 2021 era la educación virtual, con un $67.2 \%$, mientras que entre las personas con ingresos superiores a los 2,000 dólares solo un 33.6\% fue proclive a la educación a distancia. En un 53.4\%

5 Unicef, marzo de 2021. Recuperado el 11 de junio de 2021, de: https://www.unicef.org/es/ comunicados-prensa/escuelas-168-millones-ninos-llevan-casi-ano-entero-cerradas-debidocovid19\#: :text=De\%20los\%2014\%20pa\%C3\%ADses\%2C\%20las,El\%20Salvador\%2C\%20Bangladesh $\% 20 \mathrm{y} \% 20$ Bolivia. 
de los casos las personas con más recursos económicos expresaron su predilección por la educación mixta (presencial y online) frente a un $29.3 \%$ de las personas con menos de 400 dólares de ingresos.

Tabla 9

Modalidad de educativa que se debería seguir en el curso escolar 2021

\begin{tabular}{|lc|c|c|c|c|}
\hline & $\begin{array}{c}\text { Educación } \\
\text { virtual }\end{array}$ & $\begin{array}{c}\text { Educación } \\
\text { presencial }\end{array}$ & $\begin{array}{c}\text { Educación } \\
\text { mixta }\end{array}$ & $\begin{array}{c}\text { No Sabe / } \\
\text { No Responde }\end{array}$ & Pearson $\chi^{2}$ \\
& $33.6 \%$ & $11.9 \%$ & $53.4 \%$ & $1.1 \%$ & 0.05 \\
\hline Más de 2,000 & $49.1 \%$ & $4.7 \%$ & $44.3 \%$ & $1.9 \%$ \\
\hline De 400 a 1,000 & $53.8 \%$ & $1.9 \%$ & $42.3 \%$ & $1.9 \%$ & \\
\hline Menos de 400 & $67.2 \%$ & $3.4 \%$ & $29.3 \%$ & $0.0 \%$ & \\
\hline
\end{tabular}

Fuente: Elaboración propia a través de datos de la Encuesta sobre la educación durante la pandemia. Febrero 2021. CIEPS.

Paradójicamente las personas con peores recursos digitales son las más proclives a una educación exclusivamente digital frente a las personas que cuentan con mejores recursos digitales que son las más menos favorables a una educación únicamente virtual. La mayoría de encuestados con ingresos de 400 dólares, un 43.3\%, conectan a sus hijos/as a las clases virtuales exclusivamente por medio de celular o smartphone mientras que los encuestados con ingresos superiores a 2,000 solo un $10 \%$ conectan a sus hijos/as a las clases virtuales por medio del dispositivo telefónico en exclusiva. Por otra parte, entre los encuestados con 2,000 dólares o más, un 64.8\% conecta a sus hijos/as exclusivamente por medio de una computadora o laptop, mientras que en el caso de los encuestados con ingresos inferiores a los 400 dólares solo una exigua minoría del $3.6 \%$ se conecta por medio de computadora o laptop en exclusiva.

En definitiva, los resultados de estas encuestas nos dejan un diagnóstico en el que los más expuestos al virus y los que cuentan con unos dispositivos digitales menos adecuados para la educación online son los menos predispuestos a vacunarse y son los menos proclives a abrir las escuelas. Y, de forma contra intuitiva, los más desahogados económicamente, que poseen mejores dispositivos de conexión y están menos expuestos al virus, son los más proclives a abrir las escuelas y están más predispuestos a vacunarse. 
Este diagnóstico nos podría llevar a elaborar una teoría de la acción racional reduccionista y esquemática en la cual las personas con menos recursos son irracionales y las personas más acaudaladas son las más racionales, es decir el capital económico es lo que aporta sensatez a los actores sociales. El problema de este enfoque es que no toma en consideración las condiciones sociales de los diferentes actores.

Otra forma de interpretar estos resultados es que la clave de estas actitudes es el diferencial acceso a bienes y recursos básicos, es decir que tras esta dualidad opinativa se esconde una dualidad social que no se puede explicar exclusivamente por los mayores o menores ingresos. Posiblemente esta serie de cautelas y desconfianzas tiene que ver con que estos sectores sociales más vulnerables tienen una percepción de desprotección basados en un deficiente acceso a unos mínimos de seguridad sanitaria que comprende una insuficiente prevención, un deficiente seguimiento y una defectuosa atención, y desconfian de que las autoridades se las vayan a aportar.

Estas cautelas y desconfianzas de los más vulnerables se entienden mejor al constatar que un millón de habitantes no están incorporados en el sistema nacional de seguridad social, es decir uno de cada cuatro (INEC 2019c). Los hospitales adolecen de una baja ratio de camas por cada mil habitantes. En marzo de 2018 había 9,004 camas hospitalarias en todo el país, unas 2.2 camas por cada mil habitantes, mientras que en las comarcas indígenas la proporción es tan solo de 1.1 camas por cada mil (INEC 2019d). La recomendación mínima de la OMS es de tres camas por cada mil habitantes. Estar por debajo de esta recomendación genera graves riesgos para el sistema sanitario como se ha podido constatar durante la pandemia del Covid-19 en 2020. Según el índice de seguridad sanitaria global, Panamá ocupa el puesto 68 entre 195 países de acuerdo a su capacidad de responder a emergencias sanitarias. Esto se debe mayormente a un desempeño por debajo del promedio mundial en seguir normas internacionales de cooperación y financiamiento y un muy deficiente sistema de comunicación con las personas que laboran en el sistema sanitario sobre prevención y atención en épocas de crisis (GHS 2019).

Los sectores con menos recursos económicos no están preparados para hacer frente a los riesgos sanitarios de la pandemia a diferencia de otros grupos de ingresos que sí los pueden asumir porque tienen un acceso a bienes y servicios privados que pueden aminorar los riesgos.

\section{Conclusiones}

Panamá es un país que ha crecido tanto las últimas décadas que se le llegó a llamar el Singapur latinoamericano, pero este crecimiento no pudo revertir las altas tasas de desigualdad. Con el estallido de la pandemia las desigualdades estructurales presentes en el Istmo se han hecho más evidentes, seis meses antes de la pandemia en la Encuesta de Ciudadanía y Derechos, una encuesta presencial de opinión pública realizada por el CIEPS, se hicieron manifiestas la alta desconfianza y la dualidad social, dos fenómenos fuertemente asociados a la desigualdad. Esta dualidad se 
materializa en que el grupo de trabajadores universitarios tienda a depender en mayor medida de su capacidad de crédito, ahorros y propiedades, y del acceso al mercado laboral, mientras que los grupos con peores accesos al trabajo, al crédito, y a las propiedades y menos nivel educativo dependen en mayor medida de los amigos y los familiares, de los contactos con líderes políticos o religiosos, todo ello en un país en que los trabajadores con nivel universitario son minoría.

En la Encuesta de Ciudadanía y Derecho se manifiestan considerables diferencias de opinión entre estos dos grupos ya que las personas con estudios primarios apuestan con mayor intensidad por un modelo exclusivamente público para resolver sus necesidades sanitarias, educativas y de vivienda frente al grupo con estudios universitarios que, prefieren una provisión público-privada de los servicios educativos, sanitarios y de vivienda lo que da claves para entender las dificultades para articular soluciones país a la crisis del Coronavirus.

A partir de las encuestas de opinión realizadas por CIEPS durante el 2020, es posible distinguir diferencias en las posiciones de los distintos grupos identificados. De forma contraintuitiva los encuestados de menores ingresos y peores medios de acceso a internet para las clases virtuales manifestaron el mayor grado de desconfianza hacia las vacunas y el menor grado de receptividad a abrir las escuelas, apostando por la educación a distancia, y contrariamente de lo que se pudiera pensar, el grupo de ingreso con mayores recursos económicos y mejores dispositivos de conexión para las clases son los que más confian en las vacunas, y los más proclives a abrir las escuelas.

Frente a esta paradoja en vez de una interpretación que estigmatice a las personas vulnerables es importante analizar las dispares condiciones sociales que muestran un enorme diferencial en el acceso a los diferentes bienes y servicios básicos.

En el caso de la pandemia tiene una especial relevancia el dispar acceso a la salud, en definitiva, la gran desigualdad sanitaria. Esta desigualdad genera un alto nivel de desprotección social que dificulta abordar una serie de retos fundamentales para superar la crisis del coronavirus como son la inmunización de la población para llegar a "la inmunidad de rebaño", la reapertura económica que redunde en la mejora de las condiciones de vida de la población, la restitución de los servicios públicos afectados, y la progresiva recuperación de los derechos políticos y civiles suspendidos durante el estado de emergencia. 


\section{Referencias}

Astudillo, J.; Fernández, M.; Garcimartin, C. (2019). La desigualdad en Panamá: su carácter territorial y el papel de las inversiones públicas. Banco Interamericano de Desarrollo. Nota Técnica No IDB-TN-1703. Recuperado el 30 de abril de 2021 de: https:// publications.iadb.org/es/la-desigualdad-de-panama-su-caracter-territorial-y-el-papelde-las-inversiones-publicas.

Bárcena, A. (2020). Enfrentar los efectos cada vez mayores del COVID 19 para una reactivación con igualdad: nuevas proyecciones. 15 de julio de 2020. Observatorio Covid-19 en América Latina y El Caribe. Impacto económico y social. CEPAL, 2020, pp. 19. Recuperado el 20 de abril de 2021 de: https://www.cepal.org/es/presentaciones/enfrentar-efectoscada-vez-mayores-covid-19-reactivacion-igualdad-nuevas-proyecciones.

Bjornskov, C. (2007). "Determinants of generalized trust: A cross-country comparison". Public Choise (130).

Bourdieu, P. (1997). Capital cultural, escuela y espacio social. Madrid: Siglo XXI Editores.

Burke, P. (2010). Hibridismo cultural. Akal. Madrid.

Castillero Calvo,A. (2018). "Los grupos de poder en la colonia". En Antología histórica: artículos, ensayos y conferencias. Panamá: Senacyt.

Cecchini, S.; Holz, R. y Rodríguez Mojica, A. (2020). La matriz de la desigualdad en Panamá. Serie Políticas Sociales 236, CEPAL. ISSN 1680-8983.

Centro Internacional de Estudios Políticos y Sociales (CIEPS, 2021). Estudios de opinión pública. Recuperado el 30 de abril de 2021 de: https://cieps.org.pa/estudios-de-opinion/.

Coleman, J. (1988). "Social capital in the creation of human capital". American Journal of Sociology, $\mathrm{N}^{\circ} 94$.

Comité de Alto Nivel de Seguridad Hídrica (2016). Plan Nacional de Seguridad Hídrica 20152050: Agua para Todos. Panamá, República de Panamá.

Comisión Económica para América Latina y el Caribe (CEPAL, 2019). Panorama social de América Latina 2019. Naciones Unidas, Santiago de Chile.

Conniff, M. L. y Bigler, G. E. (2019). Panamá moderno: De territorio ocupado a centro de las Américas. Panamá: Ciudad de Saber.

Cuartas Ricaurte, J. (2016). ¿Desigualdad y pobreza como determinantes de la confianza generalizada? Análisis de datos panel. (U.D. Andes Ed.) Desarrollo y Sociedad. 
De León, N. (2019). PISA: mitos y realidades (parte II). En el blog de CIEPS. Recuperado el 30 de abril de 2021 de: https://cieps.org.pa/pisa-mitos-realidades-y-aprendizajesparte-ii/.

García Canclini, N. (2012). Culturas híbridas: estrategias para entrar y salir de la modernidad. Paidós Buenos Aires.

GHS Index (2019). Recuperado el 30 de abril de 2021 de: https://www.ghsindex.org/ country/panama/.

Knack, S. y Keefer, P. (1997). "Does social capital have an economic pay off? A cross-country investigation". Quaterly Journal of Economics, 112.

Instituto Nacional de Estadística y Censo (INEC). 2019a. Panamá en cifras 2013-2017. Panamá. Contraloría General de la República.

INEC 2019b. Producto Interno Bruto en la República, según provincia, Años 2016-2017. Panamá. Contraloría General de la República.

INEC (2019c). Estadísticas Sociales. Recuperado el 1 de mayo de 2021 de: http://www.inec. gob.pa/archivos/A231POBLACIÓN\%20PROTEGIDA_2018.pdf.

INEC (2019d). Camas en las instalaciones de salud en la República, por provincia, y comarca indígena y ciudad de Panamá. Recuperado el 30 de abril de 2021 de: https://www.inec.gob.pa/ avance/Default2.aspx?ID_CIFRAS=23\&ID_CATEGORIA=5\&ID_IDIOMA=1 .

INEC (2020). Empleo informal en la República: Encuesta de mercado laboral telefónica, septiembre de 2020. Recuperado el 21 de abril de 2021, de: https://www.inec.gob.pa/publicaciones/ Default3.aspx?ID_PUBLICACION=1037\&ID_CATEGORIA $=5 \&$ ID_ SUBCATEGORIA $=38$

Lambert, J. (1967). Latin America: Social Structure and Political Institutions. Berkley and Los Angeles: University of California Press.

Ministerio de Economía y Finanzas (MEF, 2017). Pobreza y Desigualdad en Panamá. Mapas a nivel de Distritos y Corregimientos:Año 2015. Panamá: Ministerio de Economía y Finanzas. Dirección de Análisis Económico y Social y Banco Mundial.

Organización para la Cooperación y el Desarrollo Económico (OCDE, 2018). Estudio multidimensional de Panamá.Volumen 2. Análisis detallado y recomendaciones. Mensajes principales, caminos de desarrollo. Recuperado el 4 de junio de 2021 de: https://www. oecd.org/development/mdcr/countries/panama/Estudio-Multidimensional-dePanama-Volumen-2-Espanol-Mensajes-principales.pdf

Rivera Cusicanqui, S. y De Souza Santos, B. (2013). "Conversas do mundo". Proyecto Alice CES/UC. Bolivia, Recuperado el 4 de junio de 2021 de: https://www.youtube.com/ watch?v $=$ xjgHfSrLnpU. 
Schleicher, A. (2019). PISA 2018: Insights and Interpretations. OECD 2019. Recuperado el 22 de abril de 2021 de: https://www.oecd.org/pisa/PISA\%202018\%20Insights\%20 and\%20Interpretations\%20FINAL\%20PDF.pdf,

Seligson, M. (1972) La tesis de la sociedad dual: Una reexaminación del caso de Costa Rica. Universidad de Pittsburgh. Publicado originalmente en la revista "Social Forces" en septiembre de 1972, vol. 51, No. 1, pp 91-98.

Therborn, G. (2013). Los campos de exterminio de la desigualdad. Buenos Aires: Fondo de Cultura Económica.

Tumín, M. (1971). Social Class and Social Change in Puerto Rico (segunda edición). Indianápolis: Bobs-Merrill.

Urabarri, R. (2020). Coronavirus y desigualdad digital. En el blog de CIEPS. Recuperado el 30 de abril de 2021 de: https://cieps.org.pa/coronavirus_y_desigualdad_digital/.

Recibido: 2 de mayo de 2021

Aceptado: 02 de julio de 2021 
